\title{
New Trend of Surgery in Locally Advanced Esophageal Squamous Cell Carcinoma Patients
}

\author{
Tatcha Rerkrak, M.D., Somkiat Sunpaweravong, M.D.
}

Department of Surgery, Faculty of Medicine, Prince of Songkla University, Hat Yai, Songkhla 90110, Thailand.

Received 2 January $2021 \bullet$ Revised 15 February 2021 • Accepted 1 March 2021 • Published online 1 June 2021

\begin{abstract}
:
The treatment of locally advanced esophageal squamous cell carcinoma is still controversial. Although, preoperative chemoradiation, followed by esophagectomy is the standard treatment, morbidity and patients' quality of life problems after an esophagectomy are still considerable. Since a good pathologic complete response rate in patients after preoperative chemoradiation, a wait and see policy (with active surveillance) has been introduced as a new alternative approach after chemoradiation. Active surveillance involves imaging and biopsy evaluations in patients after chemoradiation to detect residual or recurrent tumors. If there are no residual tumors, observation is considered. Surgery is reserved for patients who present with residual tumors or locoregional recurrence after surveillance to achieve complete resection (salvage esophagectomy). Based on evidence from recent studies, surveillance with salvage esophagectomy is a treatment option for locally advanced esophageal squamous cell carcinoma patients achieving a clinical complete response after chemoradiation.
\end{abstract}

Keywords: chemotherapy, esophageal cancer, radiation, squamous cell carcinoma, surgery

\section{Contact: Somkiat Sunpaweravong, M.D.}

Department of Surgery, Faculty of Medicine, Prince of Songkla University,

Hat Yai, Songkhla 90110, Thailand.

E-mail: susomkia@medicine.psu.ac.th

This is an open access article under the CC BY-NC-ND license

(http://www.jhsmr.org/index.php/jhsmr/about/editorialPolicies\#openAccessPolicy).

J Health Sci Med Res 2022;40(1):111-116 doi: $10.31584 /$ jhsmr.2021817 www.jhsmr.org 


\section{Introduction}

Esophageal cancer is an aggressive disease. The Global Cancer Observatory reported that in 2018 esophageal cancer ranked $7^{\text {th }}$ in incidence $(572,000$ new cases), and $6^{\text {th }}$ in mortality overall (509,000 deaths) worldwide. ${ }^{1}$ The $2^{\text {nd }}$ most common histologic subtypes are squamous cell carcinoma and adenocarcinoma. There are also geographic variations in esophageal cancer incidence and etiology (Table 1). ${ }^{2}$

Generally, most esophageal patients present with dysphagia, which implies locally advanced disease. After initial staging, precise tumor, node involvement, metastasis staging is conducted, based on the American Joint Committee on Cancer $8^{\text {th }}$ edition system. ${ }^{3}$ Locally advanced esophageal squamous cell carcinoma (ESCC) are defined as clinical stage cT2, N0 (high risk lesions), cT1b-cT2, N+ or cT3-cT4a, and any $\mathrm{N}^{4}$

Treatment of locally advanced ESCC is still challenging, due to the normal course of the disease and the complicated anatomy around the disease site. This leads to high morbidity and rates of recurrence, affecting survival and quality of life. Surgery, chemotherapy and radiation therapy are still the current mainstays of treatment.

\section{Overview of ESCC treatment}

The National Comprehensive Cancer Network (NCCN) guidelines version 4.2020 for esophageal and esophagogastric junction cancer recommends the following treatment options in medically fit patients; according to TNM classification: ${ }^{3,4}$

\section{Early stage:}

-pTis: endoscopic resection (with or without ablationadditional ablation if there is multifocal carcinoma in situ) or ablation alone

-pT1a: endoscopic resection with or without additional ablation (for pTis and pT1a, esophagectomy may be indicated if nodular disease)

-pT1b, N0: esophagectomy

-cT1b-cT2, N0: esophagectomy

${ }^{*} \mathrm{CT} 2$, NO (low risk lesion; tumor $<3 \mathrm{~cm}$, well differentiated)

\section{Locally advanced stage:}

-cT2, N0 (high risk lesion; lymphovascular invasion, tumorb $\geq 3 \mathrm{~cm}$, poorly differentiated) or cT1b-cT2, N+ or cT3-cT4a, any $\mathrm{N}$ : in this stage, patients with cervical ESCC will proceed to definitive concurrent chemoradiation. Whereas; thoracic ESCC will proceed to preoperative concurrent chemoradiation, followed by esophagectomy.

-cT4b (unresectable) and patients who decline surgery: definitive chemoradiation or chemotherapy alone in the setting of invasion of the trachea, great vessels or heart.

Table 1 Geographic variations between esophageal squamous cell carcinoma and adenocarcinoma

\begin{tabular}{lll}
\hline Variable & Squamous cell carcinoma & Adenocarcinoma \\
\hline Geographic areas & Asia & North America \\
Risk factors & Africa & Europe \\
& Smoking & Gastroesophageal reflux disease \\
& Alcohol drinking & Excess body weight \\
& Betel quid chewing & \\
& Very hot drinking & \\
& Low socioeconomic status
\end{tabular}




\section{Advanced stage:}

Unresectable locally advanced, recurrent or metastatic disease: systemic therapy or palliative care

\section{Response assessment}

The assessment of response should be conducted 5 to 8 weeks after complete preoperative chemoradiation by fluorodeoxyglucose (FDG)-positron emission tomography (PET)-computerized tomography (CT) (preferred) or FDGPET scans, chest and abdominal CT scanning with contrast along with upper gastrointestinal endoscopy and biopsy. ${ }^{4}$ A recent meta-analysis highlighted the poor accuracy of endoscopic biopsies, CT, and 18F-FDG-PET-CT, when used as lone modalities for assessing residual disease, following preoperative chemoradiation to treat esophageal cancer. ${ }^{5,6}$

After assessment, patients are classified into 3 groups:

1. No evidence of disease: proceed to esophagectomy or surveillance.

2. Persistent local disease: proceed to esophagectomy.

3. Metastatic or unresectable disease: proceed to palliative management.

\section{Preoperative chemoradiation vs. surgery alone}

In the past, esophagectomy alone was the main treatment for ESCC, but this resulted in high surgical morbidity and diminished quality of life. Additionally, this was also associated with higher rates of local recurrence in approximately half of the patients in less than 3 years. The main risk factors of recurrence are invasion depth and lymph node metastasis. ${ }^{7,8}$

Preoperative chemoradiation has both disease free and overall survival benefits compared with only surgery in patients with locally advanced ESCC. The principles of this treatment are to treat micrometastases, downstage the disease and facilitate a curative $(\mathrm{R} 0)$ resection.

In 2010, a meta-analysis found preoperative chemoradiation was superior in terms of survival benefit over only surgery alone in over 4,000 patients with resectable esophageal carcinoma; with a preoperative chemoradiation mortality hazard ratio $(\mathrm{HR})$ being $0.80(0.68-0.93$; $p-v a l u e=$ 0.004). ${ }^{9}$

The Chemoradiotherapy Oesophageal Cancer followed by Surgery Study (CROSS trial), a large randomized trial in 2012, randomized 368 patients with resectable esophageal or esophagogastric junction cancer (T1, N1, M0 or $\mathrm{T} 2-3, \mathrm{~N} 0-1, \mathrm{M} 0$ ) into surgery alone and preoperative chemoradiation followed by surgery groups. The majority of patients $(75.0 \%)$ had an adenocarcinoma. The study found median overall survival rates of 49.4 months in the chemoradiation-surgery group compared with 24.0 months in the surgery only group ( $p$-value=0.003). In subgroup analysis, the study found complete pathological response (pCR) in 18/37 ESCC patients (49.0\%). There were no significant differences in 30-day mortality between the groups. ${ }^{10}$

A large randomized trial called: the Neoadjuvant Chemoradiotherapy followed by Surgery versus Surgery alone for Locally Advanced Squamous Cell Carcinoma of the Esophagus (NEOCRTEC5010) study, compared surgery only vs. preoperative chemoradiation in 451 patients with resectable thoracic ESCC (T1-4, N1, M0 or T4, N0, M0). The study found a pathological complete response rate of $43.2 \%$, with a median overall survival of 100.1 vs 66.5 months ( $p-v a l u e=0.025$ ), and disease-free survival of 100.1 versus 41.7 months ( $p$-value $<0.001$ ), mortality of $2.2 \%$ and $0.4 \%(p-$ value $=0.212)$, and a higher $\mathrm{R} 0$ resection rate (98.4\% vs $91.2 \%)$ in the preoperative chemoradiation group than the surgery group $(p-v a l u e=0.002) .{ }^{11}$

Currently, the standard treatment for locally advanced squamous cell carcinoma of the esophagus is preoperative 
concurrent chemoradiation, followed by esophagectomy. ${ }^{4}$ However, since many studies, as noted above, have found a good response as assessed by pCR for preoperative chemoradiation, this opens the concept of no surgery in patients who achieve clinical complete response.

\section{Definitive chemoradiation vs. preoperative chemoradiation-surgery}

No survival benefit was found for further surgery in a study by the Federation Francophone de Cancerologie Digestive (FFCD 9102) trial. This study randomly assigned 259 patients who had responded to preoperative chemoradiation (T3, N0-1, M0 esophageal cancer patients received 2 cycles of fluorouracil and cisplatin and concomitant radiation) continuation of chemoradiation (three cycles of fluorouracil/cisplatin and radiation) and surgery groups. The continuation of definitive chemoradiation was deemed as being the same as preoperative chemoradiation-surgery. After two years, the chemoradiation-surgery group had a $34.0 \%$ survival rate (median survival 17.7 months) versus a $40.0 \%$ survival rate (median 19.3 months) in the definitive chemoradiation group $(p-v a l u e=0.440)$. The mortality rates were $9.3 \%$ in the chemoradiation-surgery group vs. $0.8 \%$ in the definitive chemoradiation group $(p-v a l u e=0.002) .{ }^{12}$ Thus, with this study showing no benefit for additional surgery, following chemoradiation compared with definitive chemoradiation, in patients with locally advanced squamous cell carcinoma of the esophagus the concept of surveillance alone following definitive chemoradiation alone becomes open.

\section{Wait and see vs surgery}

The concept of surveillance is to observe patients, who achieved a clinical complete response after preoperative or definitive chemoradiation, and then move on to surgery if locoregional recurrence later occurs.
A meta-analysis in 2019 showed postoperative salvage esophagectomy complications after definitive chemoradiation; anastomosis leakage in $18.2 \%$ of the patients, pulmonary complications in $30.2 \%$ and $30-$ day mortality of $3.2 \%$, figures which are comparable to preoperative chemoradiation-surgery. ${ }^{13}$

Another prospective study (JCOG0909) found clinical complete response after definitive chemoradiation of $59.0 \%$. Salvage surgery was performed in $32.0 \%$ of the patients, and the mortality rate was only $4.0 \%$; with the 3-years overall survival being $74.2 \%$. These results show that salvage surgery for locoregional failure patients, after definitive chemoradiation, is a promising option. ${ }^{14}$

In the 2019, Esophagectomy in Complete Responders to Preoperative Chemoradiotherapy for Esophageal Squamous Cell Carcinoma (ESOPRESSO) trial, 38 (44.2\%) patients who had achieved clinical complete response (cCR) after chemoradiation (T3-T4a, Nany, M0 or Tany, N+, M0 ESCC received chemoradiation) were then randomized into surgery and close observation alone groups. Survival was similar in both groups, of whom neither group achieved median overall survival ( $p$-value=0.560); although there was non-significantly, slightly better disease-free survival in the surgery group $(p-v a l u e=0.262) .{ }^{15}$ However, the study did indicate that observation with salvage surgery might be a reasonable choice in achieving $\mathrm{CCR}$ in ESCC patients after chemoradiation.

A recent prospective cohort study in esophageal cancer patients treated with preoperative chemoradiation was conducted to examine patients' preferences regarding active surveillance versus esophagectomy. In this discretechoice experiment, the patients were asked to choose between various treatment strategies, taking into account factors such as short-term/ong-term quality of life and survival. Interestingly, they found that many patients were willing to trade off substantial 5-years survival to achieve a reduction in the risk of esophagectomy. ${ }^{16}$ 


\section{Surveillance}

Since surveillance is an alternative option in locally advanced ESCC patients who have clinical complete response after chemoradiation, the accuracy of diagnostic tools for surveillance is concerned with residual tumors after chemoradiation which may be missed. The NCCN guidelines recommend clinical follow up every 3-6 months for 2 years. This is then followed by every 6-12 months for 3-5 years, and then annually thereafter. ${ }^{4}$ Although, there is no widely accepted protocol for follow up imaging serial endoscopic with biopsy and CT scan or PET-CT every 3 months $\times 2,6$ months $\times 3$, then annually is popular. ${ }^{13}$ This is because most locoregional recurrences, after definitive chemoradiation, occur within 12-24 months. ${ }^{17}$

\section{Conclusion}

Outcomes of locally advanced ESCC could be improved by combined treatments. This is dependent on the location of the cancer; for example in the cervical area definitive chemoradiation, or in the thoracic area preoperative chemoradiation; followed by surgery. Due to good responses of chemoradiation, and the desire to avoid an unnecessary esophagectomy, observation with salvage surgery in patients achieving clinical complete response might become a trend in the future.

\section{Acknowledgement}

The authors would like to thank the International Affairs Department, for assistance with the English of this manuscript.

\section{Conflict of interest}

None

\section{References}

1. Bray F, Ferlay J, Soerjomataram I, Siegel RL, Torre LA, Jemal A. Global cancer statistics 2018: GLOBOCAN estimates of incidence and mortality worldwide for 36 cancers in 185 countries. CA Cancer J Clin 2018;68:394-424.
2. Huang FL, Yu SJ. Esophageal cancer: Risk factors, genetic association, and treatment. Asian J Surg 2018;41:210-5.

3. Rice TW, Kelsen D, Blackstone EH, Ishwaran H, Patil DT, Bass AJ, et al. Esophagus and esophagogastric junction. In: Amin MA, Greene FL, Edge S, Schilsky RL, Gaspar LE, editors. AJCC cancer staging manual. $8^{\text {th }}$ ed. New York: Springer; 2017; p.185-202.

4. National Comprehensive Cancer Network. Esophageal and esophagogastric junction cancers, NCCN guidelines version 4. 2020 [homepage on the Internet]. Pennsylvania: NCCN; 2020 [cited 2020 Dec 1]. Available from: www.nccn.org

5. Eyck BM, Onstenk BD, Noordman BJ, Nieboer D, Spaander MCW, Valkema R, et al. Accuracy of detecting residual disease after neoadjuvant chemoradiotherapy for esophageal cancer: a systematic review and meta-analysis. Ann Surg 2020;271: 245-56.

6. de Gouw DJJM, Klarenbeek BR, Driessen M, Bouwense SAW, van Workum F, Fütterer JJ, et al. Detecting pathological complete response in esophageal cancer after neoadjuvant therapy based on imaging techniques: a diagnostic systematic review and meta-analysis. J Thorac Oncol 2019;14:1156-71.

7. Chen G, Wang Z, Liu XY, Liu FY. Recurrence pattern of squamous cell carcinoma in the middle thoracic esophagus after modified Ivor-Lewis esophagectomy. World J Surg 2007;31:1107-14.

8. Nakagawa S, Kanda T, Kosugi S, Ohashi M, Suzuki T, Hatakeyama K. Recurrence pattern of squamous cell carcinoma of the thoracic esophagus after extended radical esophagectomy with three-field lymphadenectomy. J Am Coll Surg 2004;198: 205-11.

9. Sjoquist KM, Burmeister BH, Smithers BM, Zalcberg JR, Simes RJ, Barbour A, et al. Survival after neoadjuvant chemotherapy or chemoradiotherapy for resectable oesophageal carcinoma: an updated meta-analysis. Lancet Oncol 2011;12:681-92.

10. van Hagen P, Hulshof MCCM, van Lanschot JJB, Steyerberg EW, van Berge Henegouwen MI, Wijnhoven BPL, et al. Preoperative chemoradiotherapy for esophageal or junctional cancer. N Engl J Med 2012;366:2074-84.

11. Yang H, Liu H, Chen Y, Zhu C, Fang W, Yu Z, et al. Neoadjuvant chemoradiotherapy followed by surgery versus surgery alone for locally advanced squamous cell carcinoma of the esophagus (NEOCRTEC5010): a phase III multicenter, randomized, open-label clinical trial. J Clin Oncol 2018;36: 2796-803. 
12. Bedenne L, Michel P, Bouché O, Milan C, Mariette C, Conroy $\mathrm{T}$, et al. Chemoradiation followed by surgery compared with chemoradiation alone in squamous cancer of the esophagus: FFCD 9102. J Clin Oncol 2007;25:1160-8.

13. Faiz Z, Dijksterhuis WPM, Burgerhof JGM, Muijs CT, Mul VEM, Wijnhoven BPL, et al. A meta-analysis on salvage surgery as a potentially curative procedure in patients with isolated local recurrent or persistent esophageal cancer after chemoradiotherapy. Eur J Surg Oncol 2019;45:931-40.

14. Ito $\mathrm{Y}$, Takeuchi H, Ogawa G, Kato K, Onozawa M, Minashi $\mathrm{K}$, et al. A single-arm confirmatory study of definitive chemoradiotherapy (dCRT) including salvage treatment in patients (pts) with clinical (c) stage II/II esophageal carcinoma (EC) (JCOG0909) [abstract]. J Clin Oncol 2018;36(Suppl 15): S4051.
15. Park SR, Yoon DH, Kim JH, Kim YH, Kim HR, Lee HJ, et al. A randomized phase III trial on the role of esophagectomy in complete responders to preoperative chemoradiotherapy for esophageal squamous cell carcinoma (ESOPRESSO). Anticancer Res 2019;39:5123-33.

16. Noordman BJ, de Bekker-Grob EW, Coene PPLO, van der Harst E, Lagarde SM, Shapiro J, et al. Patients' preferences for treatment after neoadjuvant chemoradiotherapy for oesophageal cancer. Br J Surg 2018;105:1630-38.

17. Versteijne E, van Laarhoven HW, van Hooft JE, van Os RM, Geijsen ED, van Berge Henegouwen MI, et al. Definitive chemoradiation for patients with inoperable and/or unresectable esophageal cancer: locoregional recurrence pattern. Dis Esophagus 2015;28:453-9. 